\title{
BIFURCATION IN ROTATIONAL SPECTRA OF NONLINEAR $\mathrm{AB}_{2}$ MOLECULES
}

\author{
Igor N. Kozin" \\ Institute of Applied Physics, \\ Uljanov Street 46, \\ 603600 Nizhnii Novgorod, Russia \\ and \\ I.M. Pavlichenkov² \\ Russian National Center "Kurchatov Institute", \\ 123182 Moscow, Russia
}

\footnotetext{
${ }^{1}$ E-mail: nik@appl.sci-nnov.ru
} 


\begin{abstract}
A classical microscopic theory of rovibrational motion at high angular momenta in symmetrical non-linear molecules $\mathrm{AB}_{2}$ is derived within the framework of small oscillations near the stationary states of a rotating molecule. The full-dimensional analysis including stretching vibrations has confirmed the existence of the bifurcation predicted previously by means of the rigid-bender model [see B.I. Zhilinskii and I.M. Pavlichenkov, Opt. Spectrosk. (USSR) 64, 413-414 (1988)]. The formation of fourfold energy clusters resulting from the bifurcation has been experimentally verified for $\mathrm{H}_{2} \mathrm{Se}$ and it has been demonstrated in fully-dimensional quantum mechanical calculations carried out with the MORBID computer program.

We show in the present work that apart from the level clustering, the bifurcation produces physically important effects including molecular symmetry-breaking and a transition from the normal mode to the local mode limit for the stretching vibrations due to rovibrational interaction. The application of the present theory with realistic molecular potentials to the $\mathrm{H}_{2} \mathrm{Te}, \mathrm{H}_{2} \mathrm{Se}$ and $\mathrm{H}_{2} \mathrm{~S}$ hydrides results in predictions of the bifurcation points very close to those calculated previously. However for the lighter $\mathrm{H}_{2} \mathrm{O}$ molecule we find that the bifurcation occurs at higher values of the total angular momentum than obtained in previous estimations. The present work shows it to be very unlikely that the bifurcation in $\mathrm{H}_{2} \mathrm{O}$ will lead to clustering of energy levels. This result is in agreement with recent variational calculations.
\end{abstract}




\section{INTRODUCTION}

Energy level clustering in molecules is very interesting phenomenon, which has been studied for

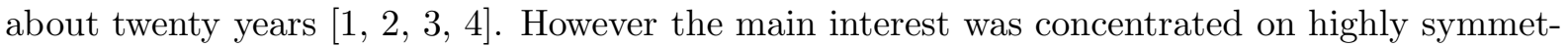
rical molecules like tetrahydrides. The possibility of fourfold cluster formation in the upper part of rotational multiplets of symmetrical non-linear triatomic molecules has been predicted in the theoretical work by one of the authors and B.I. Zhilinskii [5. In that work the classical rovibrational dynamics of an asymmetric top molecule was investigated in the rigid-bender model [6]. It has been shown that with the increasing of the angular momentum quantum number $J$ the local precession around the axis with the smallest moment of inertia changes to delocalized precession around two equivalent axes at certain critical value $J_{c}$. This phenomenon can be refered to as a bifurcation. It manifests itself in the softening of the precessional mode, i.e., in the decreasing of the spacing between the upper levels of the rotational multiplets when $J$ passes through the critical value $J_{c}$. The noticeable softening of the precessional modes in the ground and $\nu_{2}$ vibrational states of $\mathrm{H}_{2} \mathrm{O}$ and $\mathrm{H}_{2} \mathrm{~S}$ was interpreted as a possible bifurcation in this molecules [5, 7]. More precise numerical calculations based on the quantum Hamiltonian of the rigid-bender model were carried out soon [8, 9]. They demonstrated the formation of fourfold energy clusters for $J$ greater that $J_{c}$. The microscopic theory of bifurcations developed in Ref. [5] allowed the estimation of the critical angular momentum $J_{c}$ which determines the region of cluster formation. It was found in Refs. [7, 10] that $J_{c}$ decreases with increasing mass of the central atom $\mathrm{X}$ in the $\mathrm{H}_{2} \mathrm{X}$ molecule. This fact has been used in the experimental study of "level clustering" in the rotational spectrum of $\mathrm{H}_{2} \mathrm{Se}$ [11, 12]. It was established that the energy levels in the vibrational ground state of $\mathrm{H}_{2} \mathrm{Se}$ form groups of four quasi-degenerate levels at the top of the $J$-multiplets when the value of the total angular momentum $J$ exceeds the critical value $J_{c}$. This phenomenon is very unusual for the rotational spectrum of a rigid asymmetric top.

Soon afterwards the MORBID program has been successfully applied to the cluster analyses of the spectra of $\mathrm{H}_{2} \mathrm{Se}$ [13, 14], $\mathrm{H}_{2} \mathrm{~S}$ [15] and $\mathrm{H}_{2} \mathrm{Te}$ [16]. The MORBID program is a variational approach allowing the calculation of energy levels of the total rovibrational Hamiltonian of a molecule in an isolated electronic state [17]. MORBID calculations based on fitted or ab initio molecular potentials showed good agreement with known experimental data and predicted the formation of fourfold clusters in highly excited rotational states of $\mathrm{H}_{2} \mathrm{Se}, \mathrm{H}_{2} \mathrm{~S}$ and $\mathrm{H}_{2} \mathrm{Te}$. The MORBID calculations were also in good agreement with pure classical, semiclassical and model quantum mechanical estimations of the critical angular momentum $J_{c}$ for these three molecules. However preliminary calculations [18] of the rotational levels up to $J=42$ in the ground vibrational state of water molecule using the MORBID program with optimized potentials [19] did not show evident fourfold clusters. Recently Polyansky et al. have reported a new optimized water potential [20]. Their calculations up to $J=35$ for the ground vibrational state reproduce very well the available experimental energy levels but the authors concluded that the "rotational energy level structure in water is at least of a very different nature than the fourfold cluster structures observed for $\mathrm{H}_{2} \mathrm{Se}$ and calculated for $\mathrm{H}_{2} \mathrm{~S}, \mathrm{H}_{2} \mathrm{Se}$ and $\mathrm{H}_{2} \mathrm{Te}$ " [20]. These two facts are in disaccord with all estimations of $J_{c}$ for the water molecule: $J_{c}=27-28$ in Ref. [5], $J_{c}=26$ in Ref. [8] and with the calculations in Ref. [21], which predict quasi-degeneracy of the levels $J_{J, 0}, J_{J, 1}, J_{J-1,1}$, and $J_{J-1,2}$ for $J>32$.

This extensive calculations show that the role of stretching vibrations in the bifurcation phenomenon is not clear enough. The rigid-bender model used in the early microscopic theory [5] assumes the bond lengths to be fixed at their equilibrium values. This theory cannot also answer the question if a bifurcation exists in the rotational bands of excited vibrational states. This deficiency is remedied in the present paper, which uses the fully-dimensional description of rovibrational motion in a triatomic molecule, the stretching vibrations being included. The analysis of the classical equations of motion is based on the study of small harmonic oscillations

near stationary states of the system. Four-dimensional oscillations can be separated into slow precessional and fast vibrational motion. The approach of the present work differs from the stan- 
dard adiabatic approximation by Born and Oppenheimer in that it considers the rovibrational motion to take place near the stationary states of a fast uniformly rotating molecule instead of having them take place near the equilibrium state of a non-rotating molecule. The difference has very important consequences. First, the precessional motion of an $\mathrm{AB}_{2}$ molecule around the axis with minimal moment of inertia becomes unstable at the bifurcation point $J_{c}$. For higher angular momenta, the fast uniform rotation of a molecule takes place around two axes situated in the molecular plane between the axes of maximal and intermediate moments of inertia. Owing to molecular symmetry, these two axes are equivalent. The delocalized precession around them may result in fourfold level clustering. This effect is the spectroscopic manifestation of the bifurcation. Another consequence is the asymmetrical deformation of the $\mathrm{AB}_{2}$ molecule by centrifugal forces, whose anisotropic action on the B-atoms causes one $\mathrm{A}-\mathrm{B}$ bond length to be longer than the other. This symmetry breaking of the molecular configuration in its turn changes the vibrational dynamics in such a way that the normal stretching modes $\nu_{1}$ and $\nu_{3}$ transform to the local vibrations either of two $\mathrm{A}-\mathrm{B}$ bonds. It is important to note that the transition from normal mode to local mode vibrations is caused in our case by rovibrational interaction and not by the anharmonicity of the vibrations as in customary local mode theory (see, for example, the review article [22]). The developed theory allows to understand the relationship of local mode vibrations to a cluster formation in rotational spectra obtained by a model consideration in Ref. 23.

The classical method developed here has been applied to the hydrides $\mathrm{H}_{2} \mathrm{O}, \mathrm{H}_{2} \mathrm{~S}, \mathrm{H}_{2} \mathrm{Se}$, and $\mathrm{H}_{2}$ Te to demonstrate that it produces consistency with purely quantum mechanical calculations for quantities like the critical angular momentum, the bond length distortions and the bending angle of the rotating molecule. The results obtained for the $\mathrm{H}_{2} \mathrm{Te}, \mathrm{H}_{2} \mathrm{Se}, \mathrm{H}_{2} \mathrm{~S}$ molecules are very close to those obtained previously, but for the lighter $\mathrm{H}_{2} \mathrm{O}$ molecule the bifurcation is found to occur at $J$ values significantly higher than those obtained in previous estimations. The most probable explanation of this is the neglect of the stretching vibrations in the earlier models. Furthermore, our analysis shows also that the stretching vibrations result in drastic reduction of the potential barrier, which separates the two regions of a delocalized precession. Thus it is very unlikely that the bifurcation in the vibrational ground state of $\mathrm{H}_{2} \mathrm{O}$ can result in an observable fourfold cluster structure. The clustering of levels cannot therefore be the ultimate criteria of the bifurcation.

\section{CLASSICAL EQUATIONS OF ROVIBRATIONAL MOTION FOR AN $\mathrm{AB}_{2}$ MOLECULE}

We begin with the classical rovibrational Hamiltonian which can be derived following the method of Wilson, Decius, and Cross [24]. Our treatment differs from theirs in that we do not use normal coordinates to have the possibility of considering the large displacements from the equilibrium configuration. With the standard choice of Euler angles [24] as rotational coordinates, the classical kinetic rovibrational energy in a rotating coordinate system with the origin in the center of mass of molecule has the form

$$
T=\frac{1}{2} \sum_{i j} I_{i j} \omega_{i} \omega_{j}+\sum_{i} \omega_{i} g_{i}+\frac{1}{2} \sum_{\alpha i} m_{\alpha} v_{\alpha i}^{2},
$$

where the indices $i$ and $j$ run through the axes $x, y$ and $z$ of this coordinate system, $\mathbf{r}_{\alpha}\left(x_{\alpha}, y_{\alpha}, z_{\alpha}\right)$ is the position vector of nucleus $\alpha$ with mass $m_{\alpha}, \mathbf{v}_{\alpha}$ is its velocity, and $\mathbf{g}=\sum_{\alpha} m_{\alpha}\left(\mathbf{r}_{\alpha} \times \mathbf{v}_{\alpha}\right)$. $I_{i j}$ is a matrix element of the inertia tensor, and $\omega_{i}$ are the projections of an angular velocity on the axes $x, y$ and $z$ :

$$
\begin{aligned}
\omega_{x} & =\dot{\theta} \sin \chi-\dot{\varphi} \sin \theta \cos \chi \\
\omega_{y} & =\dot{\theta} \cos \chi+\dot{\varphi} \sin \theta \sin \chi \\
\omega_{z} & =\dot{\varphi} \cos \theta+\dot{\chi}
\end{aligned}
$$


We can further introduce the total angular momentum vector $\mathbf{J}$ with projections

$$
\begin{aligned}
& J_{x}=p_{\theta} \sin \chi-p_{\varphi} \csc \theta \cos \chi+p_{\chi} \cot \theta \cos \chi \\
& J_{y}=p_{\theta} \cos \chi+p_{\varphi} \csc \theta \sin \chi-p_{\chi} \cot \theta \sin \chi \\
& J_{z}=p_{\chi}
\end{aligned}
$$

which is related to the angular velocity projections as follows [24]

$$
J_{i}-g_{i}=\sum_{j} I_{i j} \omega_{j}
$$

The inversion of Eq. (4) leads to

$$
\omega_{j}=\sum_{i} \mu_{i j}\left(J_{i}-g_{i}\right)
$$

where $\left\{\mu_{i j}\right\}$ is the matrix inverse to $\left\{I_{i j}\right\}$. As a result the kinetic energy takes the form

$$
T=\frac{1}{2} \sum_{i j}\left(J_{i} J_{j}-g_{i} g_{j}\right) \mu_{i j}+\frac{1}{2} \sum_{\alpha i} m_{\alpha} v_{\alpha i}^{2} .
$$

We consider a triatomic molecule $\mathrm{B}_{1} \mathrm{~A}_{2} \mathrm{~B}_{3}$ with nuclear masses $m_{1}=m_{3}=m$ and $m_{2}=M$. To define the rotating (molecule fixed) axis system we can use one of the methods suggested by Sutcliffe and Tennyson [25]. Let the molecule be placed in the $(x z)$ plane, i.e., $y_{i}=0$. Then we define the $x$-axis to be parallel to the bisector of the bond angle $\alpha=\mathrm{B}_{1} \widehat{\mathrm{A}_{2}} \mathrm{~B}_{3}$. The axis directions are chosen so that $z$-axis points from $\mathrm{B}_{3}$ to $\mathrm{B}_{1}, x$-axis points from the centre of the mass to $\mathrm{A}_{2}$ and the coordinate system is right-handed. It is natural to introduce three internal coordinates: the $\mathrm{A}_{2}-\mathrm{B}_{1}$ distance $r_{1} \equiv q_{1}$, the angle $\alpha \equiv q_{2}$ and the $\mathrm{A}_{2}-\mathrm{B}_{3}$ distance $r_{3} \equiv q_{3}$. These internal coordinates are connected with the cartesian coordinates $x y z$ as follows

$$
\begin{aligned}
& x_{1}=\frac{m r_{3}-(M+m) r_{1}}{M+2 m} \cos \frac{\alpha}{2}, \\
& x_{2}=\frac{m\left(r_{1}+r_{3}\right)}{M+2 m} \cos \frac{\alpha}{2}, \\
& x_{3}=\frac{m r_{1}-r_{3}(M+m)}{M+2 m} \sin \frac{\alpha}{2}, \\
& z_{1}=\frac{(M+m) r_{1}+r_{3} m}{M+2 m} \sin \frac{\alpha}{2}, \\
& z_{2}=-\frac{m\left(r_{1}-r_{3}\right)}{M+2 m} \sin \frac{\alpha}{2}, \\
& z_{3}=-\frac{(M+m) r_{3}+r_{1} m}{M+2 m} \sin \frac{\alpha}{2} .
\end{aligned}
$$

With a molecule-fixed axis system defined in this way, the non-zero components of the inertiatensor can be written as

$$
\begin{aligned}
I_{x z} & =\frac{m(M+m)}{2(M+2 m)}\left(r_{1}^{2}-r_{3}^{2}\right) \sin \alpha, \\
I_{x x} & =\frac{m}{M+2 m}\left[M\left(r_{1}^{2}+r_{3}^{2}\right)+m\left(r_{1}+r_{3}\right)^{2}\right] \sin ^{2} \frac{\alpha}{2}, \\
I_{z z} & =\frac{m}{M+2 m}\left[M\left(r_{1}^{2}+r_{3}^{2}\right)+m\left(r_{1}-r_{3}\right)^{2}\right] \cos ^{2} \frac{\alpha}{2}, \\
I_{y y} & =\frac{m(M+m)}{M+2 m}\left(r_{1}^{2}+r_{3}^{2}\right)-\frac{2 m^{2}}{M+2 m} r_{1} r_{3} \cos \alpha,
\end{aligned}
$$

and the elements of the inverse matrix are 


$$
\begin{aligned}
\mu_{y y} & =1 / I_{y y}, \\
\mu_{x x} & =I_{z z} /\left(I_{z z} I_{x x}-I_{x z}^{2}\right), \\
\mu_{x z} & =I_{x z} /\left(I_{x z}^{2}-I_{z z} I_{x x}\right), \\
\mu_{z z} & =I_{x x} /\left(I_{z z} I_{x x}-I_{x z}^{2}\right) .
\end{aligned}
$$

With the introduced internal coordinates the kinetic energy (6) can be transformed to the form

$$
T=\frac{1}{2} \sum_{i j} J_{i} J_{j} \mu_{i j}+\frac{1}{2} \sum_{\nu \nu^{\prime}} c_{\nu \nu^{\prime}} \dot{q}_{\nu} \dot{q}_{\nu^{\prime}},
$$

where $i$ and $j$ again assume the values $x, y, z$, and $\nu$ and $\nu^{\prime}$ run through $1,2,3$. The $c_{\nu \nu^{\prime}}$ coefficients have the form $c_{\nu \nu^{\prime}}=a_{\nu \nu^{\prime}}-\sum_{i j} G_{i \nu} G_{j \nu^{\prime}} \mu_{i j}$, but for a planar molecule they can be simplified to $c_{\nu \nu^{\prime}}=a_{\nu \nu^{\prime}}-G_{y \nu} G_{y \nu^{\prime}} \mu_{y y}$. The matrix elements $a_{\nu \nu^{\prime}}$ depend on internal coordinates as follows

$$
\begin{aligned}
& a_{11}=a_{33}=\frac{m(M+m)}{M+2 m}, \\
& a_{22}=\frac{m}{4(M+2 m)}\left[(M+m)\left(r_{1}^{2}+r_{3}^{2}\right)+2 m r_{1} r_{3} \cos \alpha\right], \\
& a_{12}=a_{21}=\frac{m^{2}}{2(M+2 m)} r_{3} \sin \alpha, \\
& a_{23}=a_{32}=\frac{m^{2}}{2(M+2 m)} r_{1} \sin \alpha, \\
& a_{13}=a_{31}=-\frac{m^{2}}{M+2 m} \cos \alpha,
\end{aligned}
$$

and three $G_{y \nu}$ are written as

$$
\begin{aligned}
G_{y 1} & =-\frac{m^{2}}{2 m+M} r_{3} \sin \alpha, \\
G_{y 2} & =\frac{m(m+M)}{2(2 m+M)}\left(r_{1}^{2}-r_{3}^{2}\right), \\
G_{y 3} & =\frac{m^{2}}{2 m+M} r_{1} \sin \alpha .
\end{aligned}
$$

By introducing conjugate momenta $p_{\nu}=\partial T / \partial \dot{q}_{\nu}$ we can write the kinetic energy in the form

$$
T=\frac{1}{2} \sum_{i j} J_{i} J_{j} \mu_{i j}+\frac{1}{2} \sum_{\nu \nu^{\prime}} b_{\nu \nu^{\prime}} p_{\nu} p_{\nu^{\prime}}
$$

where $\left\{b_{\nu \nu^{\prime}}\right\}=\left\{c_{\nu \nu^{\prime}}\right\}^{-1}$ assuming $\operatorname{Det}\left\{c_{\nu \nu^{\prime}}\right\} \neq 0$. Finally the rovibrational Hamiltonian of the symmetrical $\mathrm{AB}_{2}$ molecule is expressed by

$$
H=\frac{1}{2} \sum_{i j} J_{i} J_{j} \mu_{i j}+\frac{1}{2} \sum_{\nu \nu^{\prime}} b_{\nu \nu^{\prime}} p_{\nu} p_{\nu^{\prime}}+V\left(r_{1}, \alpha, r_{3}\right),
$$

where $V$ is the nuclear potential, which is symmetrical with respect to permutations of identical nuclei.

The obtained Hamiltonian allows to write Hamilton's equations of motion

$$
\begin{aligned}
\dot{q}_{\nu} & =\sum_{\nu^{\prime}} b_{\nu^{\prime} \nu} p_{\nu^{\prime}} \\
\dot{p}_{\nu} & =-\frac{1}{2} \sum_{i j} \frac{\partial \mu_{i j}}{\partial q_{\nu}} J_{i} J_{j}-\frac{1}{2} \sum_{\nu^{\prime} \nu^{\prime \prime}} \frac{\partial b_{\nu^{\prime} \nu^{\prime \prime}}}{\partial q_{\nu}} p_{\nu^{\prime}} p_{\nu^{\prime \prime}}-\frac{\partial V}{\partial q_{\nu}} \\
\dot{J}_{i} & =\sum_{i} e_{i j k} J_{j} \mu_{k l} J_{l} .
\end{aligned}
$$


The equation (17) is derived by using the Poisson bracket $\left\{J_{i}, J_{j}\right\}=e_{i j k} J_{k}$, where $e$ is asymmetrical tensor and repeated indexes are summed. Equation (17) is equivalent to the three equations

$$
\begin{aligned}
& \dot{J}_{x}=\left[\mu_{x z} J_{x}+\left(\mu_{z z}-\mu_{y y}\right) J_{z}\right] J_{y}, \\
& \dot{J}_{y}=\left(\mu_{x x}-\mu_{z z}\right) J_{x} J_{z}+\mu_{x z}\left(J_{z}^{2}-J_{x}^{2}\right), \\
& \dot{J}_{z}=\left[-\mu_{x z} J_{z}+\left(\mu_{y y}-\mu_{x x}\right) J_{x}\right] J_{y} .
\end{aligned}
$$

They are not independent since $\mathbf{J}^{2}$ is an integral of motion.

\section{PRECESSIONAL AND VIBRATIONAL MOTIONS OF AN ROTATING $\mathrm{AB}_{2}$ MOLECULE}

Our analysis of the equations of motion is based on the study of rotation-vibrational motion near stationary states. In a stationary state, the time derivatives given in the left hand sides of Eqs. (15 17) are equal to zero. Hence, in a stationary state Eq. (15) takes the form

$$
\sum_{\nu^{\prime}} b_{\nu^{\prime}} p_{\nu^{\prime}}=0
$$

and it is easy to see that $p_{\nu}=0$, since the determinant of the $\left\{b_{\nu \nu^{\prime}}\right\}$ matrix is non-zero as mentioned above. Now the right part of Eq. (16) can be written in the form

$$
\frac{\partial V_{\text {eff }}}{\partial q_{\nu}}=0
$$

where the effective potential $V_{\text {eff }}$ is given by

$$
V_{\text {eff }}=\frac{1}{2} \sum_{i j} \mu_{i j} J_{i} J_{j}+V \text {. }
$$

The three Eqs. (22) determine the configuration (i.e., the values of $r_{s 1}, \alpha_{s}$, and $r_{s 3}$ ) of a rotating molecule in a stationary state. The effective potential $V_{\text {eff }}$ of Eq. (23) includes the centrifugal energy, which plays a central role in the dynamics of a rotating molecule. In particular, the configuration of a rotating molecule in a stationary state is different from that of non-rotating one. We will distinguish the corresponding values by indexes $s$ (for the stationary state) and $e$ (for the non-rotating molecule in its equilibrium configuration), respectively. Although the function $\mu_{x z}$ is antisymmetrical under the permutation of the coordinates $r_{1}$ and $r_{3}$ (see Eq. (8)), the effective potential (Eq. (23)), the total Hamiltonian (Eq. (14)), and the corresponding equations of motion are invariant under the operations of the $\mathrm{C}_{2 v}(\mathrm{M})$ permutation-inversion group of the $\mathrm{AB}_{2}$ molecule [26] (see also Ref. [27]). This group includes identity operator E, the $\mathrm{P}(13)$ permutation operator of identical nuclei, the operator of inversion $\mathrm{E}^{*}$ and the product $\mathrm{P}(13) \mathrm{E}^{*}$ (see Table II).

Equations (18 20) together with the conservation law for $\mathbf{J}^{2}$ define the axis of uniform rotation of the molecule, i.e., the total angular momentum vector $\mathbf{J}_{s}$ in the molecular frame. Three projections of this vector are determined by four algebraic equations. Therefore, in a stationary state $\mathbf{J}_{s}$ cannot have an arbitrary direction. There are two types of stationary states. In three axial stationary states $S_{i}: J_{s i}= \pm J, i=x, y, z$, the molecule rotates uniformly around the $i$-axis. The molecular equilibrium configuration in the state $S_{i}$ is defined by the three equations

$$
\frac{1}{2} \frac{\partial \mu_{i i}}{\partial q_{\nu}} J^{2}+\frac{\partial V}{\partial q_{\nu}}=0
$$

with $\nu=1,2,3$. The molecule has a symmetrical configuration with $r_{s 1}=r_{s 3}=r_{s}$ in an axial stationary state $\mathrm{S}_{i}$. The energy of this state is

$$
E_{i}=\frac{1}{-} \mu_{i j}\left(r_{s}, \alpha_{s}, r_{s}\right) J^{2}+V\left(r_{s}, \alpha_{s}, r_{s}\right) .
$$


There is only one plane stationary state $S_{x z}$. In this state, the molecule rotates uniformly around one of the two equivalent axes situated in the $(x z)$ plane symmetrically relative to the $x$-axis. They form angles $\beta_{s}$ and $\left(\pi-\beta_{s}\right)$ with the $z$-axis. The configuration of the molecule and the value of $\beta_{s}$ are defined by the equations

$$
\begin{aligned}
& \frac{1}{2}\left(\frac{\partial \mu_{x x}}{\partial q_{\nu}} \sin ^{2} \beta_{s}+\frac{\partial \mu_{x z}}{\partial q_{\nu}} \sin 2 \beta_{s}+\frac{\partial \mu_{z z}}{\partial q_{\nu}} \cos ^{2} \beta_{s}\right) J^{2}+\frac{\partial V}{\partial q_{\nu}}=0, \quad \nu=1,2,3 \\
& \frac{1}{2}\left(\mu_{x x}-\mu_{z z}\right) \sin 2 \beta_{s}+\mu_{x z} \cos 2 \beta_{s}=0 .
\end{aligned}
$$

It is interesting that in the state $S_{x z}$, the molecule has an asymmetric configuration with $r_{s 1} \neq$ $r_{s 3}$. Of the two bond lengths, the one which initially forms the largest angle with the rotation axis will experience a larger elongation due to centrifugal forces, and for increasing $J$ this bond length will tend to become perpendicular to the rotation axis. When we have obtained one solution of Eqs. (26 27), the remaining three solutions can be easily obtained by using symmetry operations of the $\mathrm{C}_{2 v}(\mathrm{M})$ group from Table [1. The energy of the molecule in the $S_{x z}$ state is equal to

$$
E_{x z}=\frac{1}{2} J^{2}\left(\mu_{x x} \sin ^{2} \beta_{s}+\mu_{x z} \sin 2 \beta_{s}+\mu_{z z} \cos ^{2} \beta_{s}\right)+V\left(r_{s 1}, \alpha_{s}, r_{s 3}\right) .
$$

The analysis of stationary states shows that an axial state has higher symmetry in the $\mathrm{C}_{2 v}(\mathrm{M})$ group than the plane state. This means that transitions from axial to plane state or vice versa are accompanied by a $\mathrm{C}_{2 v}$-type bifurcation [10]. Such a bifurcation has been considered for the first time in rotational spectra of symmetrical triatomic molecules in Ref. [5]. However, with the rigid-bender model with fixed bond lengths used in that work, it was not possible to show that the bifurcation results not only in the splitting of the stationary axis and consequently in level clustering but also in molecular symmetry-breaking. Due to this latter effect, the vibrational dynamics of the rotating molecule changes as we shall see below. Table II shows the centrifugal distortions $\Delta r_{\nu}=r_{s \nu}-r_{e}$ of the bond lengths in the stationary state $S_{x z}$. The values were obtained numerically for the molecules $\mathrm{H}_{2} \mathrm{Se}$ and $\mathrm{H}_{2} \mathrm{~S}$ by using the potentials from Refs. [13] and [15], respectively. For comparison, the $\Delta r_{\nu}$ values obtained for the quantum cluster states in the upper part of the $J$-multiplets belonging to the ground vibrational state of these molecules

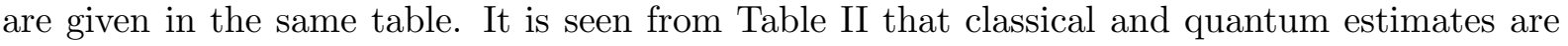
in a good agreement.

Since the derivative $\frac{\partial \mu_{z z}}{\partial \alpha}$ is positive in the stationary state $S_{z}$, the bending angle $\alpha_{s}$ decreases with increasing $J$ according to Eq. (24). After a bifurcation point, the angle $\alpha_{s}$ is stabilized at a constant value in the stationary state $S_{x z}$. In the rigid-bender model this angle is independent of $J$ for $J>J_{c}$ [5, 7] (see Fig. 1). For $\mathrm{H}_{2} \mathrm{Se}$, the rigid-bender and stretching-bender models give comparable results, but for $\mathrm{H}_{2} \mathrm{O}$ the predictions of the stretching-bender model deviate significantly from those of the rigid-bender (Fig. 国). In the stretching-bender model, the bending angle value for $\mathrm{H}_{2} \mathrm{O}$ continues to decrease significantly when $J$ is above the critical value. Hence no stabilization at a constant value takes place. This effect can be explained as a competition between centrifugal force causing the hydrogen nuclei to bring together and their mutual repulsion.

In order to follow the change of molecule rotational regimes as $J$ increases, we must investigate the stability of the stationary states. To do this we consider the linearized set of Eqs. (15 17) for small displacements of the internal coordinates $Q_{\nu}=q_{\nu}-q_{s \nu}$ and the angular momentum projections $J_{i}^{\prime}=J_{i}-J_{s i}$ from their stationary values. Let us begin with the axial stationary state $S_{y}$. The linearized equations have the form

$$
\begin{aligned}
& \sum_{\nu^{\prime}}\left(c_{\nu \nu^{\prime}} \ddot{Q}_{\nu^{\prime}}+\frac{\partial^{2} V_{\text {eff }}}{\partial q_{\nu} \partial q_{\nu^{\prime}}} Q_{\nu^{\prime}}\right)=0, \\
& \ddot{J}_{x}+J^{2}\left(\mu_{x x}-\mu_{y y}\right)\left(\mu_{z z}-\mu_{y y}\right) J_{x}=0 .
\end{aligned}
$$

The coefficients of these equations are assumed to be taken in the stationary state, i.e., for 
state. Equations (29) describe two independent harmonic motions: vibrational and precessional. The latter motion is characterized by the precession frequency

$$
\Omega_{y}=J\left[\left(\mu_{x x}-\mu_{y y}\right)\left(\mu_{z z}-\mu_{y y}\right)\right]^{1 / 2},
$$

which has the same form as for a rigid top [28]. The vibrational motion in the state $S_{y}$ does not differ from that of non-rotating molecule except in that the frequencies $\omega_{1}, \omega_{2}$, and $\omega_{3}$ of three normal modes $\nu_{1}, \nu_{2}$, and $\nu_{3}$ [24] are determined by the effective potential and the equilibrium configuration of rotating molecule. As a result they are shifted relative to those of non-rotating molecule (see below).

The linearized equations for the stationary state $S_{z}$

$$
\begin{aligned}
& \sum_{\nu^{\prime}}\left(c_{\nu \nu^{\prime}} \ddot{Q}_{\nu^{\prime}}+\frac{\partial^{2} V_{\mathrm{eff}}}{\partial q_{\nu} \partial q_{\nu^{\prime}}} Q_{\nu^{\prime}}\right)+J \frac{\partial \mu_{x z}}{\partial q_{\nu}} J_{x}=0 \\
& \ddot{J}_{x}+J^{2}\left(\mu_{z z}-\mu_{y y}\right)\left\{\left(\mu_{z z}-\mu_{x x}\right) J_{x}-J \sum_{\nu} \frac{\partial \mu_{x z}}{\partial q_{\nu}} Q_{\nu}\right\}=0
\end{aligned}
$$

describe coupled vibrational and precessional motions. It is not difficult to see that the coupling originates in the antisymmetrical variable $Q_{a}=\left(Q_{1}-Q_{3}\right) / \sqrt{2}$. Thus Eqs. (31) split into two independent parts for the variables $Q_{a}, J_{x}$ and $Q_{s}=\left(Q_{1}+Q_{3}\right) / \sqrt{2}, Q_{2}$. The equations for $Q_{s}$ and $Q_{2}$ are independent of the precessional motion and describe the $\nu_{1}$ and $\nu_{2}$ vibrational modes modified by molecular rotation. We can separate the precessional motion from the antisymmetric vibration by using the inequality $\omega_{3} \gg \Omega_{z}$ (adiabatic approximation), which is correct up to the critical point $J_{c}$ (see below). In this approximation, the frequency of molecular precession around the $z$-axis is equal to

$$
\Omega_{z}=J\left[\left(\mu_{z z}-\mu_{y y}\right)\left\{\mu_{z z}-\mu_{x x}+J^{2}\left(\frac{\partial \mu_{x z}}{\partial r_{a}}\right)^{2} / \frac{\partial^{2} V_{\text {eff }}}{\partial r_{a}^{2}}\right\}\right]^{1 / 2},
$$

where according to Eqs. (8) and (9)

$$
\mu_{z z}-\mu_{x x}=\frac{2(M+m)}{m M r_{s}^{2} \sin \alpha_{s}}\left(\frac{m}{M+m}-\cos \alpha_{s}\right) .
$$

For the hydrides considered, the equilibrium angle $\alpha_{e}$ is larger than $90^{\circ}$. So the precession frequency of Eq. (32) is real and the stationary state $S_{z}$ is stable for small values of $J$. As we saw above, the stationary angle $\alpha_{s}$ decreases with increasing $J$ due to a centrifugal distortion. So $\mu_{z z}$ approaches $\mu_{x x}$ and the precession frequency $\Omega_{z}$ vanishes when

$$
\mu_{z z}-\mu_{x x}+J_{c}^{2}\left(\frac{\partial \mu_{x z}}{\partial r_{a}}\right)^{2} / \frac{\partial^{2} V_{\mathrm{eff}}}{\partial r_{a}^{2}}=0
$$

Equations (34) and (24) (for $i=z$ ) define the critical angular momentum $J_{c}$. In the rigid-bender model, this point is determined by the bending angle

$$
\alpha_{c}=\arccos \left(\frac{m}{M+m}\right)
$$

for which $I_{x x}=I_{z z}$. The last term in Eq. (34) takes into account the non-rigidity of the bond lengths $r_{1}$ and $r_{3}$ and becomes important when $\mu_{z z}-\mu_{x x}$ is close to zero. Since the correction term is positive, Eq. (34) gives higher values of $J_{c}$ than the rigid-bender model. Clearly, the more rigid the antisymmetrical stretching mode $\nu_{3}$, the closer is $J_{c}$ to the value obtained in Ref. [7] in the rigid-bender approximation. Our numerical calculations of critical angular momentum for the set of the $\mathrm{H}_{2} \mathrm{X}$ hydrides with the potentials from Refs. 13, 15, 16] give mainly slight corrections to the $J_{c}$ values predicted previously. We obtained $J_{c}=9.3$ for $\mathrm{H}_{2} \mathrm{Te}, J_{c}=12.5$ for 
It is seen that for lighter molecules, larger discrepancies are obtained. An exception is the water molecule for which the discrepancy of the $J_{c}$ value is very significant. Using the potential from Ref. [19] $J_{c}=26.5$ was obtained with the "frozen" bond lengths (which is close to all previous estimations) and $J_{c}=35.2$ in the stretching-bender model.

For $J>J_{c}$, the stationary state $S_{z}$ corresponds to an unstable saddle-type point. It is the top of a separation barrier between two symmetrical states $S_{x z}$ which appear because of the bifurcation. The equations of a small amplitude motion near this state have the form

$$
\begin{aligned}
& \sum_{\nu^{\prime}}\left(c_{\nu \nu^{\prime}} \ddot{Q}_{\nu^{\prime}}+\frac{\partial^{2} V_{\mathrm{eff}}}{\partial q_{\nu} \partial q_{\nu^{\prime}}} Q_{\nu^{\prime}}\right)+\frac{J B_{\nu}}{\cos \beta_{s}} J_{x}^{\prime}=0, \\
& \ddot{J}_{x}^{\prime}+J^{2}\left(\mu_{z z}-\mu_{y y}+\mu_{x z} \tan \beta_{s}\right)\left(\frac{\mu_{z z}-\mu_{x x}}{\cos 2 \beta_{s}} J_{x}^{\prime}-J \cos \beta_{s} \sum_{\nu} B_{\nu} Q_{\nu}\right)=0,
\end{aligned}
$$

where the "coupling constant"

$$
B_{\nu}=\frac{1}{2} \frac{\partial\left(\mu_{x x}-\mu_{z z}\right)}{\partial q_{\nu}} \sin 2 \beta_{s}+\frac{\partial \mu_{x z}}{\partial q_{\nu}} \cos 2 \beta_{s}
$$

mixes all four motions: three vibrations and a precession. The last one can be decoupled from the vibrations in the adiabatic approximation for moderate values of $J$. In this approximation, the frequency of the precession around the $S_{x z}$ state is

$$
\Omega_{x z}=J\left[\left(\mu_{z z}-\mu_{y y}+\mu_{x z} \tan \beta_{s}\right)\left(\frac{\mu_{z z}-\mu_{x x}}{\cos 2 \beta_{s}}+\frac{J^{2}}{\Delta} \sum_{\nu, \nu^{\prime}}(-1)^{\nu-\nu^{\prime}} \Delta_{\nu \nu^{\prime}} B_{\nu} B_{\nu^{\prime}}\right)\right]^{1 / 2},
$$

where the determinant $\Delta=\operatorname{Det}\left\{\frac{\partial^{2} V_{\text {eff }}}{\partial q_{\nu} \partial q_{\nu^{\prime}}}\right\}$ and $\Delta_{\nu \nu^{\prime}}=\Delta_{\nu^{\prime} \nu}$ are the minores of this determinant. All the values in Eq. (38) are assumed to be taken in the stationary point determined by Eqs. (26) and (27). It is not difficult to show that the precession frequency $\Omega_{x z}$ vanishes in the critical point $J_{c}$.

The small amplitude motion near the state $S_{x}$ is described by Eqs. (31) when index $x$ is replaced by $z$. As a result we get in the adiabatic approximation the following expression for the precession frequency around the $x$-axis

$$
\Omega_{x}=J\left[\left(\mu_{x x}-\mu_{y y}\right)\left\{\mu_{x x}-\mu_{z z}+J^{2}\left(\frac{\partial \mu_{x z}}{\partial r_{a}}\right)^{2} / \frac{\partial^{2} V_{\mathrm{eff}}}{\partial r_{a}^{2}}\right\}\right]^{1 / 2},
$$

where all the values are taken in the stationary state $S_{x}$. The last term in the braces is a small correction. Consequently, the stability of the $S_{x}$ state is mainly defined by the value given in Eq. (33) which in turn depends on the angle $\alpha_{s}$. Since this angle is larger than $90^{\circ}$ for the molecules considered and increases with increasing $J$ because the derivative $\frac{\partial \mu_{x x}}{\partial \alpha}$ is negative, the precession frequency $\Omega_{x}$ is imaginary for reasonable values of $J$. Thus the state $S_{x}$ corresponds to an unstable saddle-type point. The $J$-dependence of its energy is defined by Eq. (25).

Equations (25) and (28) (when the transition $S_{z} \rightarrow S_{x z}$ occurs) provide a good estimation of the maximal rotation energy in $J$-multiplet. The difference between our estimations and experimental data or variational calculations for $\mathrm{H}_{2} \mathrm{Te}, \mathrm{H}_{2} \mathrm{Se}, \mathrm{H}_{2} \mathrm{~S}$ and $\mathrm{H}_{2} \mathrm{O}$ molecules does not exceed $10 \%$. This is also true for our estimations of the minimal rotational energy in the $J$-multiplet using Eq. (25) for the state $S_{y}$. The energy of the unstable stationary state $S_{x}$ corresponds to the top of the barrier responsible for the inversion doublets ( $K$-doublets) in the upper and lower parts of the $J$-multiplets [3]. Another barrier appears because of the bifurcation for angular momentum at $J>J_{c}$. It separates two degenerate maxima of the stationary state $S_{x z}$. The barrier is responsible for the twofold clustering of the inversion doublets. Its height is equal to the difference between the energies of the states $S_{z}$ and $S_{x z}$. The higher the barrier is, the more apparent is the clustering. In the $\mathrm{H}_{2} \mathrm{Te}, \mathrm{H}_{2} \mathrm{Se}$ and $\mathrm{H}_{2} \mathrm{~S}$ molecules, the height of the 
with potentials from Ref. [19] show that the height of the energy barrier increases very slowly with increasing $J$ and its value is only $15 \mathrm{~cm}^{-1}$ at $J=40$ (see Fig. 2). This value is less than the precession frequency in the $S_{x z}$ state so that such a barrier cannot result in fourfold clustering of levels. For higher $J$ values the barrier increases further to its maximal value $150 \mathrm{~cm}^{-1}$ (which is about the precession frequency at this point) and then begins to decrease. In contrary, the barrier rapidly increases with increasing $J$ for the rigid-bender model as it is seen from Fig. 2, where the dependence of the barrier height on angular momentum $J$ is plotted. It is obvious that water represents a special case in the series of $\mathrm{H}_{2} \mathrm{X}$ hydrides. Despite the bifurcation in the ground vibrational state of this molecule, no fourfold level clustering can be observed in the upper part of $J$-multiplets since the barrier is too small. This fact gives us a physical explanation to the recent numerical calculations of water rotational spectrum with highly accurate optimized potentials [18, 20], which failed to show any fourfold clusters.

The $\mathrm{C}_{2 v}$ bifurcation has to exist also in rotational energy structure of excited vibrational states so far as the adiabatic approximation $\omega \gg \Omega$ is valid and allows to separate the precession from the vibrational motion. As to the energy clustering effect, we have seen above that this problem requires separate and more careful consideration. Preliminary analysis of experimental data showed the tendency of levels to cluster in $\nu_{2}$ vibrational states of the $\mathrm{H}_{2} \mathrm{O}$ and $\mathrm{H}_{2} \mathrm{~S}$ molecules [5, 7]. The cluster phenomenon has been found in variational quantum calculations for the excited vibrational states of $\mathrm{H}_{2} \mathrm{Se}$ [13, 14, $\mathrm{H}_{2} \mathrm{~S}$ [15] and $\mathrm{H}_{2} \mathrm{Te}$ [16] and very recently observed experimentally in the $\nu_{1} / \nu_{3}$ state of $\mathrm{H}_{2} \mathrm{Se}$ [29].

Consider now in detail the molecular vibrational motion near the stationary states $S_{z}$ for $J<J_{c}$ and $S_{x z}$ for $J>J_{c}$. Four normal modes of precession-vibration motion are described by four coupled Eqs. (31) and (36) correspondingly. All these equations are not correct near the critical point $J_{c}$ where the rovibrational motion is strongly non-linear. It was shown above that the equations for the axial state $S_{z}$ (and those for the state $S_{x}$ ) can be separated into two independent pairs when symmetrized stretching coordinates are introduced. When $J=0$ the precessional motion is absent and the vibrational motion corresponds to the well-known picture of the normal vibrational modes $\nu_{1}, \nu_{2}$ and $\nu_{3}$ [24]. This limit allows us to refer to the standard notations for a rotating molecule as well, i.e., the frequency $\omega_{1}$ corresponds to the symmetrical stretching mode $\nu_{1}$, the frequency $\omega_{2}$ corresponds to the bending mode $\nu_{2}$, and the frequency $\omega_{3}$ corresponds to the antisymmetrical stretching mode $\nu_{3}$. The difference from a non-rotating molecule consists in the effective potential for vibrations and in the equilibrium configuration, which is symmetrical but differs from that of non-rotating molecule. Besides, the precessional mode with frequency $\Omega_{z}$ appears and the antisymmetrical stretching vibration mixes with the precessional motion because of a rovibrational interaction. However this mixing is very weak. This can be seen from Table III where the normalized amplitudes of four coordinates $Q_{1}, Q_{2}$, $Q_{3}$, and $Q_{4}=J_{x} / J$ involved in a normal mode oscillations are given for the $\mathrm{H}_{2}$ Se molecule at $J=10$. It is seen from the table that the mixing of different types of motions is small and the character of the vibrations is close to the standard normal mode picture [24].

The character of the oscillations changes drastically after the critical point $J_{c}$. It is impossible to split the four equations (36) describing the small amplitude motion near the stationary state $S_{x z}$ into two independent pairs because the molecular configuration is asymmetric and the uniform rotation of molecule around the axis in the $(x z)$ plane mixes the symmetrical and antisymmetrical stretching vibrations. As a result, the standard picture of normal mode vibrations, which is correct for the $S_{z}$ state, breaks down. This effect is illustrated in Table IV which shows normalized amplitudes of oscillations around the asymmetrical configuration of the state $S_{x z}$ at $J=16\left(\beta_{s}=26.08^{\circ}\right)$. It shows also that the precessional motion is mixed mostly with the bending vibration. It is worth noting that the vibrations in the mode with the frequency $\omega_{1}$ are localized predominantly on the bond $r_{1}$ and vibrations in the mode with the frequency $\omega_{3}$ are localized on the bond $r_{3}$. This feature is inherent in local mode vibrations [22]. However usually the local mode motion appears due to the strong anharmonicity as the stretching vibrations are increasingly excited. In our case the normal to local mode transition is a result of the symmetry breaking, which leads to the different force constants for two bonds and favors the local mode 
picture in the competition between kinetic and potential coupling between the local bond oscillators. Accordingly the local character of the stretching motion strengthens with increasing $J$. This follows from Table $\mathrm{V}$ which shows normalized amplitudes of oscillations around the state $S_{x z}$ at $J=40\left(\beta_{s}=42.26^{\circ}\right)$.

The classical calculations of the present work have shown that in non-linear $\mathrm{AB}_{2}$ molecules, a normal to local mode transition can be brought about by rotational excitation. Similar results have been obtained in quantum numerical calculations with the MORBID program [14]. The classical analysis of precession-vibrational motion of the present work gives a physical picture of the phenomenon. That is, the bifurcation in a ground vibrational state breaks the molecular symmetry and changes both rotational and vibrational motions of the non-linear $\mathrm{AB}_{2}$ molecule resulting in the fourfold clusters and in the normal to local mode transition. The physical reasons for these phenomena lie in the molecular symmetry and in the anisotropic centrifugal force. Therefore we should expect similar or even more interesting phenomena in the fast rotating molecules with higher symmetry.

\section{ACKNOWLEDGMENTS}

This work was supported by the Russian Fond for Fundamental Investigations (through grant N 94-02-05424-a), International Science Foundation and Russian Government (through grants MIW000, MIW300 and R8I000, R8I300). The authors are indebted to P. Jensen for critically reading the manuscript and suggesting improvements. I.N.K. is thankful to A.F. Krupnov for valuable comments on the manuscript.

\section{References}

[1] A.J. Dorney and J.K.G. Watson, J. Mol. Spectrosc. 42, 135 (1972).

[2] W.G. Harter and C.W. Patterson, J. Chem. Phys. 80, 4241 (1984) and references therein.

[3] W.G. Harter, Comp. Phys. Rep. 8, 319 (1988).

[4] D.A. Sadovskit, B.I. Zhilinskit, J.-P. Champion and G. Pierre, J. Chem. Phys. 92, 1523 (1990).

[5] B.I. Zhilinskit and I.M. Pavlichenkov, Opt. Spectrosc. (USSR) 64, 413 (1988).

[6] J.T. Hougen, P.R. Bunker and J.W.C. Johns, J. Mol. Spectrosc. 34, 136 (1970).

[7] I.M. PaVlichenkov, Fizika atomnogo jadra (Materials of XXIV-th winter school LIJaF) 67, Leningrad 1989.

[8] J. Makarewicz and J. Pyka, Mol. Phys. 68, 107 (1989); J. Makarewicz, Mol. Phys. 69, 903 (1990).

[9] J. PykA, Mol. Phys. 70, 547 (1990).

[10] I.M. Pavlichenkov, Phys. Reports 226, 175 (1993).

[11] I.N. Kozin, S.P. Belov, O.L. Polyansky and M.Yu. Tretyakov, J. Mol. Spectrosc. 152, 13 (1992).

[12] I.N. Kozin, S. Klee, P. Jensen, O.L. Polyansky and I.M. Pavlichenkov, J. Mol. Spectrosc. 158, 409 (1993).

[13] P. Jensen and I.N. Kozin, J. Mol. Spectrosc. 160, 39 (1993). 
[15] I.N. Kozin And P. Jensen, J. Mol. Spectrosc. 163, 483 (1994).

[16] P. Jensen, Yan Li, G. Hirsch, R.J. Buenker, T.J. Lee and I.N. Kozin, Chem. Phys. 190, 179 (1995).

[17] P. Jensen, J. Mol. Spectrosc., 128, 478 (1988); J. Chem. Soc. Faraday Trans. 2, 84, 1315 (1988).

[18] P. Jensen and I.N. Kozin, unpublished results.

[19] P. Jensen, J. Mol. Spectrosc., 133, 438 (1989); P. Jensen, S. Tashkun, and Vl.G. Tyuterev, J. Mol. Spectrosc., 168, 271 (1994).

[20] O.L. Polyansky, P. Jensen and J. Tennyson, J. Chem. Phys. 101, 7651 (1994).

[21] L.H. Coudert, J. Mol. Spectrosc. 154, 427 (1992).

[22] M.S. Child and L. Halonen, Adv. Chem. Phys. 57, 1 (1984).

[23] K.K. Lehmann, J. Chem. Phys. 95, 2361 (1991).

[24] E.B. Wilson, Jr., J.C. Decius and P.C. Cross, "Molecular Vibrations", McGraw-Hill Book Company, N.Y. 1955.

[25] B.T. Sutcliffe and J. Tennyson, Mol. Phys., 58, 1053 (1986); Int. J. of Quantum Chemistry, 39, 183 (1991).

[26] P.R. Bunker, "Molecular Symmetry and Spectroscopy", Academic Press, London, 1979.

[27] P. Jensen and P.R. Bunker, J. Mol. Spectrosc. 164, 315 (1994).

[28] L.D. Landau And E.M. Lifshitz, "Mechanics", Pergamon, Oxford, 1962.

[29] J.-M. Flaud, C. Camy-Peyret, H. Bürger, P. Jensen and I.N. Kozin, to be published in J. Mol. Spectrosc. 


\section{FIGURE CAPTIONS}

Figure 1: $J$-dependence of the bending angle in the stationary states with maximal rotational energy $\left(S_{z}\right.$ if $J<J_{c}$ and $S_{x z}$ if $J>J_{c}$ ) for the $\mathrm{H}_{2}$ Se and $\mathrm{H}_{2} \mathrm{O}$ molecules. The angle is displayed relative to the equilibrium value $\alpha_{e}$ for non-rotating molecule. The solid lines correspond to the stretching-bender model with the critical angular momentum $J_{c}=12.5\left(\mathrm{H}_{2} \mathrm{Se}\right)$ and $35.2\left(\mathrm{H}_{2} \mathrm{O}\right)$; the dashed lines represent the rigid-bender model $\left(J_{c}=11.4\right.$ for the $\mathrm{H}_{2}$ Se and 26.5 for the $\mathrm{H}_{2} \mathrm{O}$ molecules).

Figure 2: Value of the barrier which separates two equivalent maxima of stationary state $S_{x z}$ for water molecule calculated in the stretching-bender model (solid line) and the rigid-bender model (dashed line). 


\section{TABLE CAPTIONS}

Table I: Action of $\mathrm{C}_{2 v}(\mathrm{M})$ group operators on the internal coordinates and the projections of the total angular momentum $\mathbf{J}$.

Table II: Comparison of the molecular centrifugal distortions $\Delta r_{\nu}=r_{s \nu}-r_{e \nu}$ for the stationary state $S_{x z}$ at $J=20$ calculated in quantum and classical methods. The classical calculations correspond to molecular rotation around the stationary axis which is approximately perpendicular to the bond $r_{1}$. Indexes $s$ and $e$ stand for the molecular equilibrium configurations of rotating and non-rotating molecule correspondingly.

Table III: Normalized amplitudes of coordinate oscillations in the normal modes of precessionvibrational motion $\left(Q_{4}=J_{x} / J\right)$ around stationary state $S_{z}$ for $\mathrm{H}_{2}$ Se molecule at $J=10$.

Table IV: Normalized amplitudes of coordinate oscillations in the normal modes of precessionvibrational motion $\left(Q_{4}=J_{x}^{\prime} / J_{s z}\right)$ near the equilibrium configuration of the $\mathrm{H}_{2}$ Se molecule uniformly rotating in stationary state $S_{x z}$ at $J=16$ around the axis which is approximately perpendicular to the $r_{1}$ bond $\left(\beta_{s}=26.08^{\circ}\right)$.

Table V: Normalized amplitudes of coordinate oscillations in the normal modes of precessionvibrational motion $\left(Q_{4}=J_{x}^{\prime} / J_{s z}\right)$ near the equilibrium configuration of the $\mathrm{H}_{2}$ Se molecule uniformly rotating in stationary state $S_{x z}$ at $J=40$ around the axis which is approximately perpendicular to the $r_{1}$ bond $\left(\beta_{s}=42.26^{\circ}\right)$. 
Figure 1.
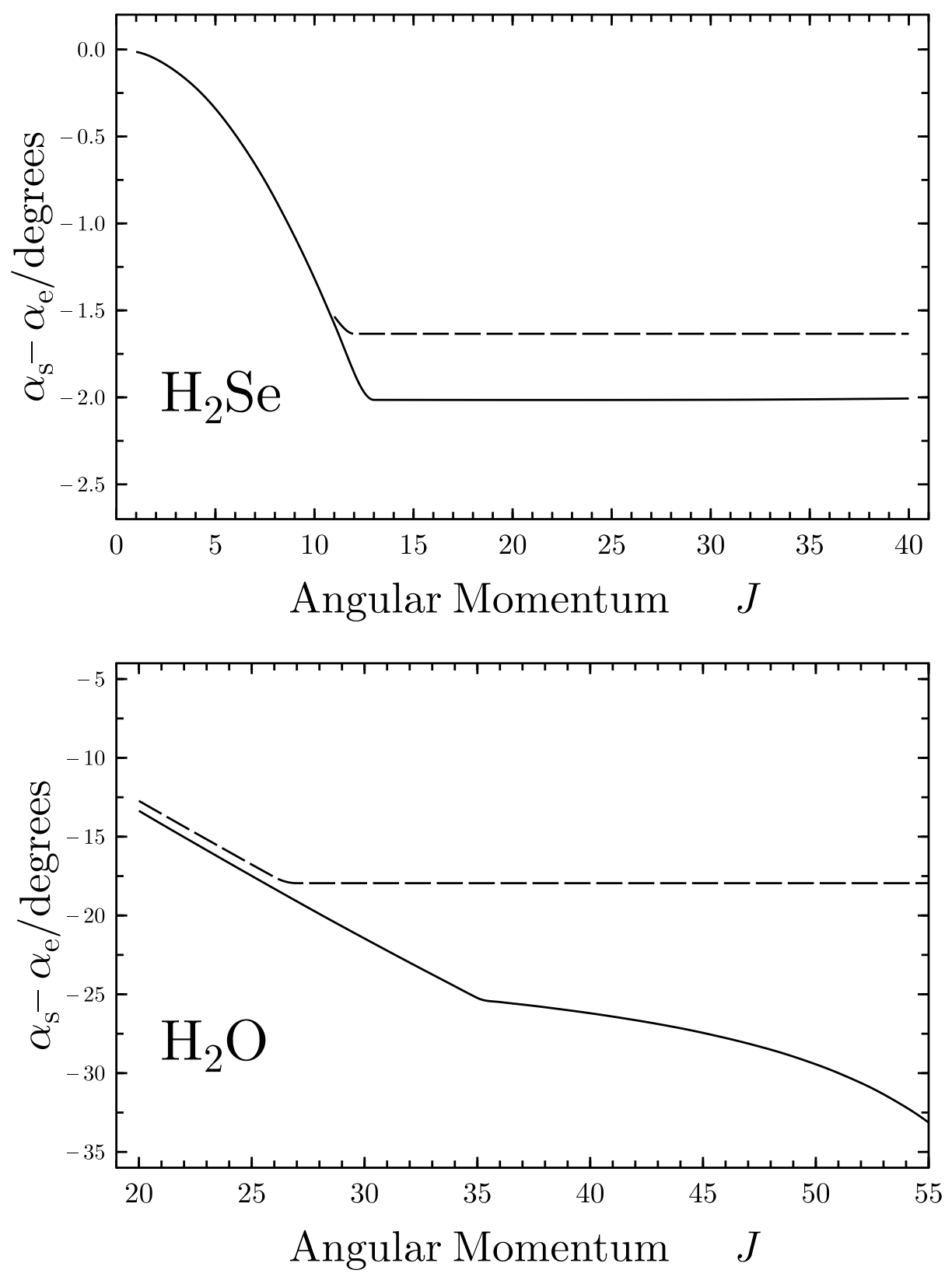
Figure 2.

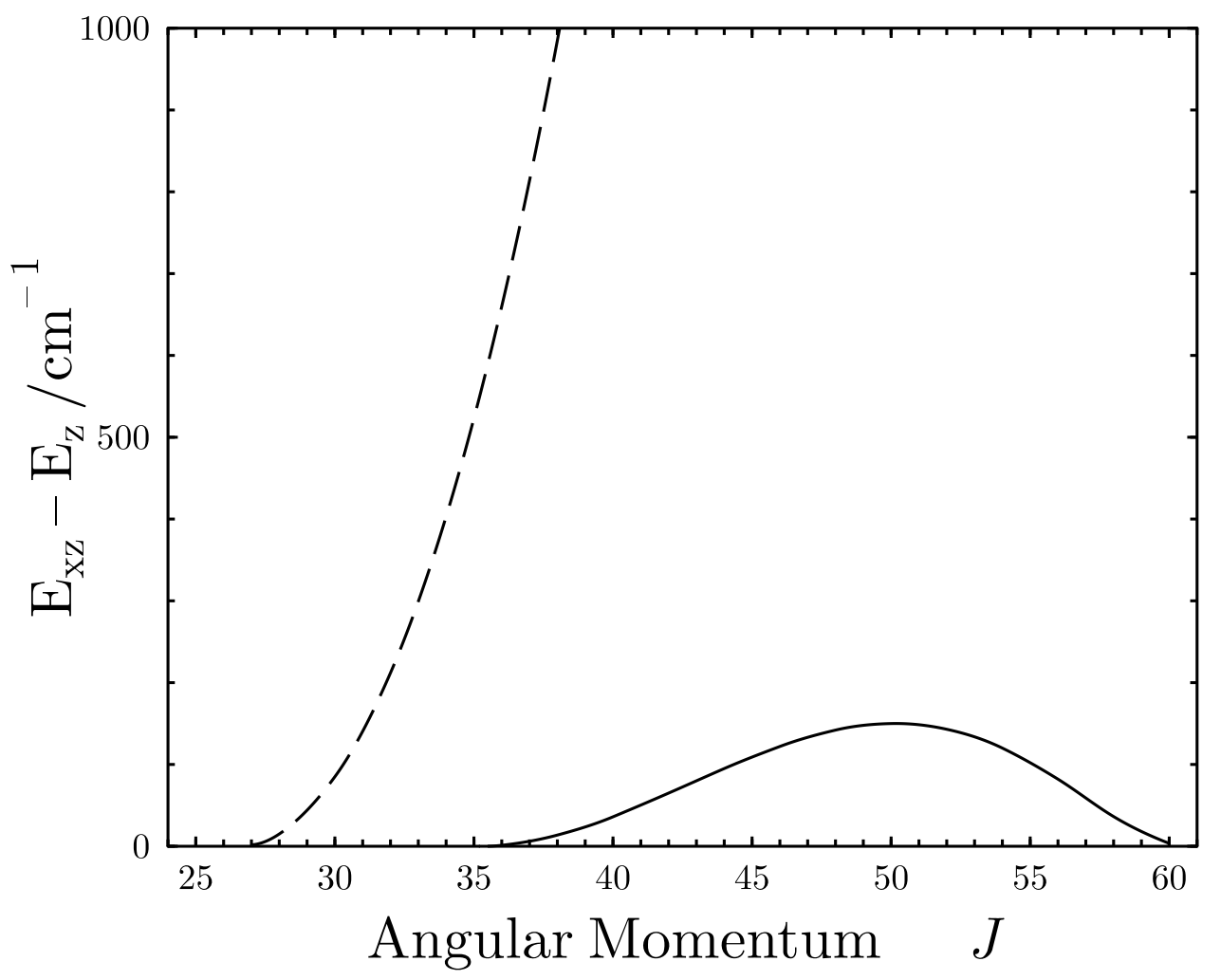


Table

\begin{tabular}{|c|cccc|}
\hline \hline & $\mathrm{E}$ & $\mathrm{P}(13)$ & $\mathrm{E}^{*}$ & $\mathrm{P}(13) \mathrm{E}^{*}$ \\
\hline$r_{1}$ & $r_{1}$ & $r_{3}$ & $r_{1}$ & $r_{3}$ \\
$r_{3}$ & $r_{3}$ & $r_{1}$ & $r_{3}$ & $r_{1}$ \\
$\alpha$ & $\alpha$ & $\alpha$ & $\alpha$ & $\alpha$ \\
$J_{x}$ & $J_{x}$ & $J_{x}$ & $-J_{x}$ & $-J_{x}$ \\
$J_{y}$ & $J_{y}$ & $-J_{y}$ & $J_{y}$ & $-J_{y}$ \\
$J_{z}$ & $J_{z}$ & $-J_{z}$ & $-J_{z}$ & $J_{z}$ \\
\hline \hline
\end{tabular}

Table II.

\begin{tabular}{|c|cc|cc|}
\hline \hline molecule & \multicolumn{2}{|c|}{ quantum estimation } & \multicolumn{2}{c|}{ classical estimation } \\
& $\Delta r_{1} / \AA$ & $\Delta r_{3} / \AA$ & $\Delta r_{1} / \AA$ & $\Delta r_{3} / \AA$ \\
\hline $\mathrm{H}_{2} \mathrm{Se}$ & $0.023^{a}$ & $0.004^{a}$ & 0.025 & 0.002 \\
$\mathrm{H}_{2} \mathrm{~S}$ & $0.024^{b}$ & $0.009^{b}$ & 0.022 & 0.010 \\
\hline \hline
\end{tabular}

$a$ - taken from Ref. 114.

$b$ _ taken from Ref. 15. 
Table III.

\begin{tabular}{|c|c|c|c|c|c|}
\hline \multirow{2}{*}{\multicolumn{2}{|c|}{$\begin{array}{c}\text { Normal } \\
\text { frequency } / \mathrm{cm}^{-1}\end{array}$}} & \multicolumn{4}{|c|}{ Normalized amplitudes } \\
\hline & & $\left(r_{1}-r_{s 1}\right) / \AA$ & $\left(\alpha-\alpha_{s}\right) / \mathrm{rad}$ & $\left(r_{3}-r_{s 3}\right) / \AA$ & $J_{x} / J$ \\
\hline$\omega_{1}=$ & 2426.4 & $1 .^{\dagger}$ & -0.032 & 1. & 0. \\
\hline$\omega_{2}=$ & 1082.3 & -0.014 & $1 .^{\dagger}$ & -0.014 & 0 . \\
\hline$\omega_{3}=$ & 2437.4 & $1 .{ }^{\dagger}$ & 0. & -1 & -0.004 \\
\hline$\Omega_{z}=$ & 15.7 & 0.004 & 0 . & -0.004 & $1 .^{\dagger}$ \\
\hline
\end{tabular}

$\dagger$ - Normalization condition $Q_{n}=1$.

Table IV.

\begin{tabular}{|cr|l|l|l|c|}
\hline \hline \multicolumn{2}{|c|}{ Normal } & \multicolumn{4}{|c|}{ Normalized amplitudes } \\
\cline { 2 - 6 } frequency $/ \mathrm{cm}^{-1}$ & $\left(r_{1}-r_{s 1}\right) / \AA$ & $\left(\alpha-\alpha_{s}\right) / \mathrm{rad}$. & $\left(r_{3}-r_{s 3}\right) / \AA$ & $\left(J_{x}-J_{s x}\right) / J_{s z}$ \\
\hline$\omega_{1}=r 2390.9$ & $1 .^{\dagger}$ & 0.018 & 0.095 & 0.003 \\
$\omega_{2}=$ & 1110.1 & -0.015 & $1 . .^{\dagger}$ & -0.013 & 0.020 \\
$\omega_{3}=$ & 2436.0 & -0.095 & 0.012 & $1 . \dagger^{\dagger}$ & -0.003 \\
$\Omega_{x z}=$ & 39.5 & 0.006 & 0.081 & -0.008 & $1 . .^{\dagger}$ \\
\hline \hline
\end{tabular}

$\dagger$ - Normalization condition $Q_{n}=1$.

Table V.

\begin{tabular}{|cr|l|l|l|l|}
\hline \hline \multicolumn{2}{|c|}{ Normal } & \multicolumn{4}{|c|}{ Normalized amplitudes } \\
\cline { 2 - 6 } frequency $/ \mathrm{cm}^{-1}$ & $\left(r_{1}-r_{s 1}\right) / \AA$ & $\left(\alpha-\alpha_{s}\right) / \mathrm{rad}$. & $\left(r_{3}-r_{s 3}\right) / \AA$ & $\left(J_{x}-J_{s x}\right) / J_{s z}$ \\
\hline$\omega_{1}=$ & 2095.8 & $1^{\dagger}$ & 0.030 & 0.015 & 0.004 \\
$\omega_{2}=$ & 1271.9 & -0.022 & $1 .^{\dagger}$ & -0.009 & 0.093 \\
$\omega_{3}=$ & 2438.0 & -0.015 & 0.003 & $1 .^{\dagger}$ & -0.002 \\
$\Omega_{x z}=$ & 211.7 & 0.000 & 0.429 & -0.009 & $1 .^{\dagger}$ \\
\hline \hline
\end{tabular}

$\dagger$ - Normalization condition $Q_{n}=1$. 